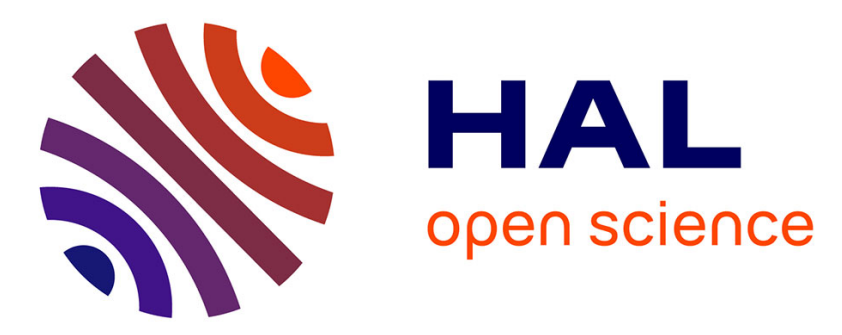

\title{
Nonresonant four wave mixing in photorefractive CdTe crystals using a picosecond parametric generator
}

Kestutis Jarašiunas, Vytautas Gudelis, Philippe Delaye, Gérald Roosen

\section{To cite this version:}

Kestutis Jarašiunas, Vytautas Gudelis, Philippe Delaye, Gérald Roosen. Nonresonant four wave mixing in photorefractive CdTe crystals using a picosecond parametric generator. Review of Scientific Instruments, 1998, 69 (11), pp.3776-3779. 10.1063/1.1149178 . hal-00673495

\section{HAL Id: hal-00673495 \\ https://hal-iogs.archives-ouvertes.fr/hal-00673495}

Submitted on 23 Feb 2012

HAL is a multi-disciplinary open access archive for the deposit and dissemination of scientific research documents, whether they are published or not. The documents may come from teaching and research institutions in France or abroad, or from public or private research centers.
L'archive ouverte pluridisciplinaire HAL, est destinée au dépôt et à la diffusion de documents scientifiques de niveau recherche, publiés ou non, émanant des établissements d'enseignement et de recherche français ou étrangers, des laboratoires publics ou privés. 


\title{
Nonresonant four-wave mixing in photorefractive CdTe crystals using a picosecond parametric generator
}

\author{
Kestutis Jarašiūnas ${ }^{\mathrm{a})}$ and Vytautas Gudelis \\ Material Science and Applied Research Institute, Vilnius University, Vilnius 2040, Lithuania \\ Philippe Delaye and Gerald Roosen \\ Laboratoire Charles Fabry de l'Institut d'Optique, Unité Associée au Centre National de la Recherche \\ Scientifique, 91403 Orsay Cedex, France
}

(Received 21 May 1998; accepted for publication 17 August 1998)

\begin{abstract}
We demonstrate that a parametrically pumped picosecond laser has enough coherence and energy to write transient phase gratings at nonresonant interaction, thus allowing a study of time-resolved carrier transport in CdTe crystals to be made. Autocorrelation trace of light diffraction efficiency on transient grating allowed us to measure a coherence length of the parametric generator. Carrier diffusion, recombination, and drift in light-created internal space-charge (SC) electric fields have been studied in vanadium or germanium doped semi-insulating CdTe crystals by nonresonant four-wave mixing technique at $940 \mathrm{~nm}$ wavelength. It was found that modification of the deep level charge state in $\mathrm{CdTe}: \mathrm{V}$ by As codoping has changed the sign of majority carriers, responsible for the creation of SC field. Dynamics of free carrier grating decay in CdTe:Ge revealed an electron-governed very fast initial grating decay which develops with time into the double-exponential hole-governed grating decay. Time-resolved transient grating technique described in this article provides a powerful tool for investigation of the role of deep traps in photorefractive semiconductors and optimization of their photoelectric properties in a required temporal and spectral range. (C) 1998 American Institute of Physics. [S0034-6748(98)02811-1]
\end{abstract}

\section{INTRODUCTION}

The search for sensitive and fast photorefractive crystals suitable for the infrared optical region is an important task due to a variety of potential applications of such materials in optical communication networks, data processing systems, or high-speed optoelectronic devices (as real-time interferometric sensors, phase-conjugated mirrors, etc.). The technique of light diffraction by a transient grating has been widely used to characterize photoelectric and nonlinear optical properties of bulk semiconductors and structures, ${ }^{1-3}$ but mainly at relatively few wavelengths, namely those emitted by appropriate solid-state lasers. High-power lasers have been used to generate nonequilibrium carriers by two-photon, interband, or deep trap assisted absorption, thus creating bipolar plasma with carrier density of $10^{17}-10^{18} \mathrm{~cm}^{-3}$. Investigation of the resonant optical nonlinearities in the vicinity of band gap (e.g., coherent interaction ${ }^{4}$ or electroabsorptive effects in quantum confined structures ${ }^{5}$ ) necessitated the use of tunable laser sources, such as Ti-sapphire or dye lasers. We will show in this article that parametric devices allow as well to write the transient gratings and do short pulse spectroscopy at nonresonant light-matter interaction. Thus a direct investigation of the wavelength dependence of midgap impurity related photoexcitation and carrier transport is possible at wavelengths well below the band gap. Moreover, application of parametric devices for investigation of optical nonlinearities would help for optimalization of photorefractive properties of the crystals in the infrared region.

${ }^{a)}$ Electronic mail: kestutis.jarasiunas@ff.vu.lt
In this article we present, to the best of our knowledge, the first studies of photorefractive semiconductors by using a parametric light source. The goal of this work was twofold: to test the coherent properties of a picosecond optical parametric generator (OPG) and perform time-resolved carrier transport measurements by the degenerate four-wave mixing (DFWM) technique in CdTe crystals at nonresonant excitation conditions, when the absorption coefficient is only few $\mathrm{cm}^{-1}$. We demonstrate that nonlinear optical measurements of light diffraction in photorefractive CdTe crystals reveal the features of carrier generation and transport, dependent both on the charge state of deep impurity (vanadium or germanium) and light-created space-charge (SC) electric field.

\section{PARAMETRIC DEVICE AND EXPERIMENTAL SETUP}

The optical scheme used to optimize pump-laser parameters as well as a configuration of an angular-tunable OPG are shown in Fig. 1. A commercial passively and actively mode-locked yttrium-aluminum-garnet (YAG) laser, operating at $10 \mathrm{~Hz}$ repetition rate was used as a master oscillator. It provided a single pulse of 27 ps duration at $\lambda=1.06 \mu \mathrm{m}$, which was spatially filtered (by lenses $\mathrm{L}_{1}, \mathrm{~L}_{2}$, and pinhole D) and amplified. The output beam had an energy of 7-8 mJ per single pulse and a beam diameter of $6-8 \mathrm{~mm}$. It was used as input beam to a potassium dihydrogen phosphate (KDP) crystal which generated the second harmonic. The $\lambda / 4$ plate (P) allowed us to adjust the polarization of the fundamental to achieve efficient frequency doubling and obtain the required polarization of the second harmonic $(\mathrm{SH})$. The $\mathrm{SH}$ 


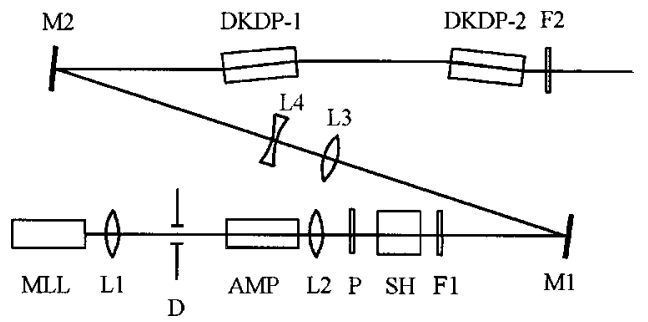

FIG. 1. The optical scheme of pump laser and a configuration of an angulartunable parametric oscillator: 1- MLL- mode-locked and $Q$-switched picosecond YAG laser, A- amplifier, SH-second harmonics crystal, L1-L4lenses, F1, F2-filters, M1, M2-mirrors, $P-\lambda / 4$ plate, DKDP-1,-2-nonlinear crystals.

beam had an energy of 4-5 $\mathrm{mJ}$ and was collimated by lenses $\mathrm{L}_{3}, \mathrm{~L}_{4}$ to a diameter of $\approx 1 \mathrm{~mm}$ and directed to two $5 \mathrm{~cm}$ long DKDP crystals: a generator and an amplifier. The OPG generated two beams (signal and idler) in two orthogonal polarizations. The total output energy of the OPG with the amplifier was found to be $0.15-0.2 \mathrm{~mJ}$, and tuning over a spectral range from 0.85 to $1.35 \mu \mathrm{m}$ was possible by angular alignment of both DKDP crystals. The output wavelength was determined by measuring the $\mathrm{SH}$ wavelength generated noncollinearly in a $\mathrm{LiIO}_{3}$ crystal.

The DFWM configuration (Fig. 2) was used to determine the temporal coherence of two interfering OPG beams by measuring light diffraction efficiency on a transient grating. ${ }^{6}$ The grating was recorded in GaAs crystal by two $s$-polarized beams at $940 \mathrm{~nm}$ by varying the delay between these beams $(\Delta t)$ over a range of a few $\mathrm{mm}$. Monitoring the diffraction efficiency of the grating by the delayed probe beam at the fixed delay time $\Delta t_{p}=2 \tau_{L}$ (here $\tau_{L}$ is duration of the OPG pulse) as the arrival time of one excite beam was varied with respect to the other, we measured the autocorrelation trace of the diffraction efficiency. The duration of the OPG pulse was measured by the $\mathrm{SH}$ autocorrelation technique ${ }^{7}$ in the $\mathrm{LiIO}_{3}$ crystal using two $p$-polarized OPG beams at $1240 \mathrm{~nm}$. Thus we used the shorter wavelength of the OPG (which is stronger absorbed in the vicinity of the absorption edge of CdTe and GaAs to record the gratings in these crystals, while the longer OPG wavelength was found strong enough to generate second harmonics in $\mathrm{LiIO}_{3}$ crystal.

Using the parametric device, we recorded transient free carrier (FC) gratings and studied carrier modulation dynam-

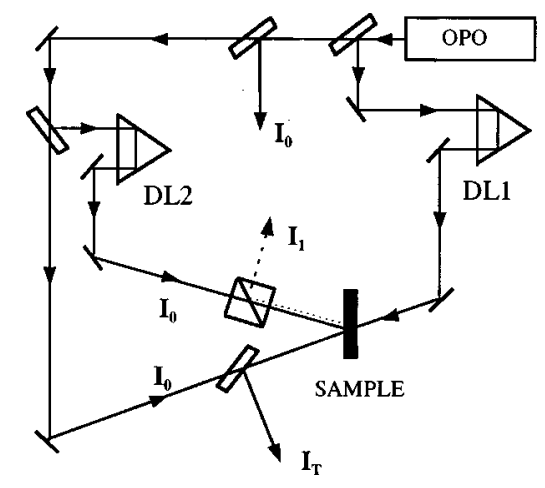

FIG. 2. The optical setup of degenerate four-wave mixing using OPG. Delay line DL2 is used to vary the delay time between the recording beams while the probe beam is delayed by DL1.

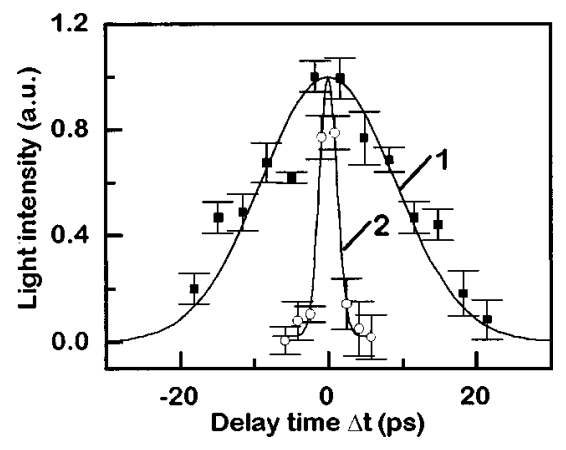

FIG. 3. Autocorrelation traces of the second harmonics generation in $\mathrm{LiIO}_{3}$ crystal (1) and of transient grating diffraction efficiency in GaAs crystals (2) for a $p$ - and $s$-polarized beams of the parametric oscillator.

ics in variously doped semi-insulating samples of CdTe in a standard configuration of $\mathrm{DFWM}^{8}$ (see Fig. 2). The beam of the OPG was divided into two parts, which intersected at an angle of $13^{\circ}$. Transient gratings with a period of $4.2 \mu \mathrm{m}$ were recorded in CdTe crystals by using $s$-polarized beams at $\lambda=940 \mathrm{~nm}$. A third beam from the OPG was delayed up to $\Delta t_{p} \leqslant 1.5 \mathrm{~ns}$ and used as a probe beam to monitor the temporal decay of the recorded grating. The energies of incident $\left(I_{0}\right)$, transmitted $\left(I_{T}\right)$, and diffracted $\left(I_{1}\right)$ signals were measured by InGaAs photodiodes and a data acquisition system, connected to a personal computer. The simple relationship between the measured signals and the refractive index modulation $\Delta n$ is given by Eq. (1)

$$
I_{1}=I_{T} \sin ^{2}(\pi \Delta n d / \lambda \cos \theta),
$$

where $d$ is the sample thickness, and $\Delta n$ follows from the Drude-Lorenz model ${ }^{1,2}$

$$
\begin{aligned}
\Delta n= & -\left[e^{2} /\left(2 n \epsilon_{0} \omega^{2}\right)\right]\left(\Delta N_{e} / m_{e}+\Delta N_{p} / m_{p}\right)\left[\omega_{g}^{2} /\left(\omega_{g}^{2}\right.\right. \\
& \left.\left.-\omega^{2}\right)\right] .
\end{aligned}
$$

Here $\Delta N_{e}$ and $\Delta N_{p}$ are the concentrations of electron and hole instantaneous modulation, $m_{e}$ and $m_{p}$ are effective masses of electrons and holes, and $\omega_{g}^{2}$ corresponds to bandgap frequency. Measuring the dynamics of free grating decay at very small refractive index modulation $\left(\Delta n \leqslant 10^{-5}\right.$, thus in Eq. (1) $\sin (\pi \Delta n d / \lambda \cos \theta) \cong(\pi \Delta n d / \lambda \cos \theta)$, and $I_{1}(t)$ $\propto[\Delta n(t)]^{2}$ ), we were able to monitor refractive index instantaneous value and get deeper insight into carrier generation, transport, and recombination mechanisms.

\section{RESULTS AND DISCUSSION}

\section{A. Parameters of the parametric device}

In Fig. 3 we present the autocorrelation traces of the $\mathrm{SH}$ intensity and of diffraction efficiency. The duration of the OPG pulse was determined by fitting the measured SH autocorrelation curve with the calculated one. The best fit to the measured half-width of $\tau_{\mathrm{SH}} \approx 20 \mathrm{ps}$ (curve 1) corresponds to the duration of a Gaussian pulse of the OPG $\tau_{L} \approx 14 \mathrm{ps,}$ which is in agreement with the well-known relationship $\tau_{\mathrm{SH}} / \tau_{L}=\sqrt{2} .^{7}$ The autocorrelation trace of the diffraction efficiency (curve 2) is much shorter than the pulse width because of the spatial modulation of the interference pattern by random phase modulation in the OPG pulse. Hence, the in- 
stantaneous amplitude of the refractive index $\Delta n(\Delta t)$ follows the temporal coherence function $\Gamma(\Delta t)$, which in a nonlinear way affects the diffraction efficiency: $\eta(\Delta t)$ $\propto[\Delta n(\Delta t)]^{2} \propto[\Gamma(\Delta t)]^{2}$. We fitted the measured coherence function $\checkmark \eta(\Delta t)$ by an exponential one $\Gamma(\Delta t)=A$ $\exp \left[-\left(\ln 2 / 2 \tau_{c}\right) t\right]$ (Ref. 2) and obtained a coherence time $2 \tau_{c} \cong 3.3 \mathrm{ps}$. The latter value leads to a coherence length $L_{c}=0.5 \mathrm{~mm}$ and to the spectral width of the OPG line $\Delta \lambda$ $=\lambda^{2} / L_{c}=1.77 \mathrm{~nm}$ at half maximum (correspondingly, $\Delta \nu$ $\left.=20 \mathrm{~cm}^{-1}\right)$.

\section{B. Dynamics of free carrier gratings in CdTe}

Using the parametric device, we investigated carrier dynamics in variously doped semi-insulating crystals of CdTe. The samples used had different deep impurities (Ge or V) and various charge states of the vanadium impurity. The presence of vanadium (or germanium) creates a deep level at $\approx E_{c}-0.8 \mathrm{eV}^{9}$ (or $E_{v}+0.73 \mathrm{eV}^{10}$ ) which stabilizes the Fermi level close to the midgap, producing the semi-insulating behavior. Charge states and their densities in CdTe:V were determined by the electron paramagnetic resonance and magnetic circular dichroism techniques ${ }^{9}$ for the used samples. The two charge states of the deep donor, namely the unionized state $V^{2+}$ and the ionized one $V^{3+}$, were present in a sample B23 with their densities of $4.7 \times 10^{15} \mathrm{~cm}^{-3}$ and 6 $\times 10^{15} \mathrm{~cm}^{-3}$, correspondingly. In another sample, B28, which was codoped with a shallow acceptor As, the ionized donor state $V^{3+}$ dominates, and its concentration was 3.5 $\times 10^{16} \mathrm{~cm}^{-3}$. The doping with Ge may lead to various effects: formation of a deep acceptor level at $E_{V}+0.73 \mathrm{eV}$ with its very high capture cross section, ${ }^{10}$ substitution of Te or/and $\mathrm{Cd}$ atoms by $\mathrm{Ge},{ }^{10}$ and inversion of a type of photoconductivity from $p$ to $n$ by increasing the dopant density. ${ }^{11}$ The absorption coefficients for the given CdTe crystals were equal to a few $\mathrm{cm}^{-1}$ at $\lambda=1.064 \mathrm{~nm}$, thus $3-5 \mathrm{~mm}$ thick samples were excited nearly homogeneously at the used OPG wavelength $\lambda=940 \mathrm{~nm}$.

In photorefractive CdTe:V crystals, nonequilibrium electrons are generated due to photoionization of deep donor state. In turn, neutralization of an ionized deep impurity state will result in hole generation. Usually the photoionization cross section for electrons exceeds the one for holes, thus photogenerated free electron concentration $N_{e}$ is higher than the hole concentration $N_{p}$. Indeed, electrons were found as the major carriers at excitation by $1.17 \mathrm{eV}$ quantum energy. ${ }^{12}$ In As-codoped CdTe, the generation of holes is expected by transitions from the valance band to the empty $V^{3+}$ states. In $\mathrm{CdTe}: \mathrm{Ge}$, a sign of photogenerated carriers may vary with the dopant density ${ }^{11}$ and quantum energy of the incident light. ${ }^{10}$

Decay of FC gratings in CdTe: $\mathrm{V}$ is shown in Fig. 4. At low excitation levels (see curve 3) a two-exponential decay process is evident. Assuming that the initial grating decay with time constant $\tau_{1}=170-190 \mathrm{ps}$ is governed by diffusion, we estimated carrier mobility $\mu_{1}=980 \pm 50 \mathrm{~cm}^{2} \mathrm{~V}^{-1} \mathrm{~s}^{-1}$ using the relationship $\tau_{1}=\tau_{D}=(\Lambda / 2 \pi)^{2}\left(e / k T \mu_{1}\right)$. The given mobility value was found to be very close to an electron mobility in undoped $\mathrm{CdTe}\left[\mu_{e}=1000 \mathrm{~cm}^{2} \mathrm{~V}^{-1} \mathrm{~s}^{-1}\right.$ (Ref.

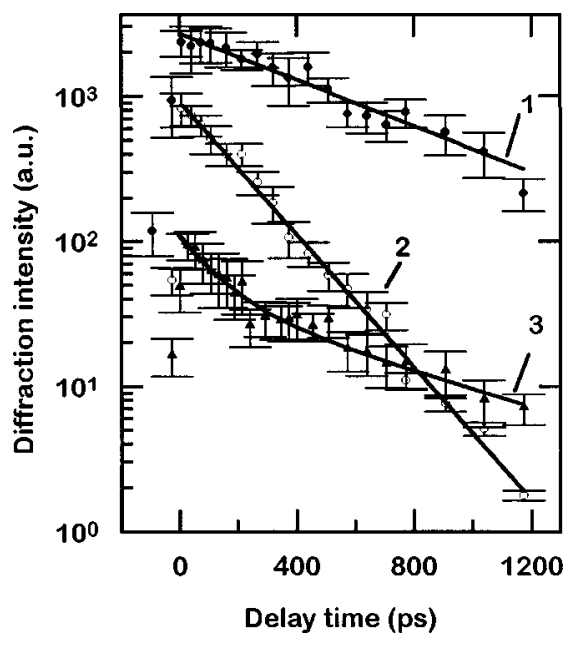

FIG. 4. Free carrier grating decay in two differently grown CdTe crystals: vanadium-doped $(1,3)$ and As-codoped (2) at excitation densities of $\sim 3$ $\mathrm{mJ} \mathrm{cm}{ }^{-2}(1,2)$ and $\sim 1 \mathrm{~mJ} \mathrm{~cm}^{-2}$ (3). The solid lines are the best fit curves to the experimental data.

13)] and pointed out that electrons are generated very efficiently at $940 \mathrm{~nm}$ wavelength. The diffusive decay of the electron grating builds up an internal SC field between the electrons, transferred to the grating minima, and the photoionized vanadium states in the grating peaks. The created SC field affects the further carrier transport: a drift of electrons in the SC field compensates the diffusive current, and grating decay slows down, revealing a second decay component with $\tau_{2}=1.2 \mathrm{~ns}$, which is linked to electron recombination time $\tau_{R} \cdot{ }^{14}$

At higher excitations only recombination-governed decay component $\tau_{R}=1.1 \mathrm{~ns}$ is seen in CdTe:V, as far the SC field is already created during the action of the laser pulse (Fig. 4, curve 1). In the latter case, the buildup time of the SC field is determined by the dielectric relaxation time $\tau_{\mathrm{di}}$ $=\epsilon \epsilon_{0} / e N_{e} \mu_{e}$ which is shorter than the duration of OPG pulse. This feature of excitation-dependent grating decay time indicates that the power of the parametric device allows us to generate high carrier concentrations.

In the As-codoped sample, where hole generation is much more effective than electrons, the hole diffusion with a time of $2 \mathrm{~ns}$ is expected to dominate in decay of FC grating at low excitation levels. At the used higher excitation levels, an exponential decay of grating with a time constant of 380 ps was found (see Fig. 4, curve 2). Thus we approach bipolar plasma regime, but still have a concentration of holes higher than that of electrons. The photogenerated carrier concentration is high enough to ensure the buildup of the SC field by the dielectric time constant (i.e., during the action of the laser pulse) which afterwards decreases at a similar rate as that of the photoexcited carrier modulation. Due to a small effective mass of electrons, their contribution to the refractive index modulation is more pronounced [see Eq. (2)], thus the values of $D=11.6 \mathrm{~cm}^{2} \mathrm{~s}^{-1}$ and mobility $\mu=460 \mathrm{~cm}^{2} \mathrm{~V}^{-1} \mathrm{~s}^{-1}$, estimated from the given above grating decay time, correspond to the diffusion of a bipolar plasma with $N_{p}>N_{e}$.

The kinetics of free carrier grating decay in Ge-doped CdTe (Fig. 5) revealed a very fast initial grating decay with $\tau_{0} \approx 60-80 \mathrm{ps}$ which develops with time into the double- 


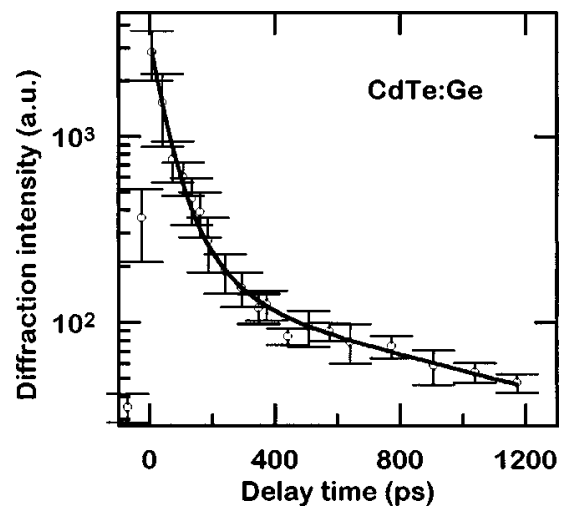

FIG. 5. Free carrier transient grating decay in Ge-doped CdTe at excitation density of $\sim 3 \mathrm{~mJ} \mathrm{~cm}^{-2}$. The solid line is the best fit curve to the experimental data.

exponential decay. The latter part is composed by the diffusion-governed decay component $\left(\tau_{1}=210 \mathrm{ps}\right)$ and deep-trap governed recombination tail $\left(\tau_{2}=1.9-2.1 \mathrm{~ns}\right)$. The essential decrease of the diffraction efficiency until the SC field is formed (what is contrary to CdTe:V at the same excitation power) indicates the overlapping of a very fast carrier trapping with diffusion: the initial part of the grating decay is even faster than the pure electron grating decay (170 ps at the given grating spacing). For the used quantum energy $h \nu=1.32 \mathrm{eV}$, the electrons can be generated from the level at $E_{v}+0.4 \mathrm{eV}^{10}$ as well as by two step transitions (via the temporarily filled acceptor at $E_{v}+0.73 \mathrm{eV}$ ), or by twophoton interband transitions. These electrons may be rapidly captured by shallow and deep donors above the Fermi level, and probably the center at $E_{c}-0.6 \mathrm{eV}$ with its huge capture cross section for electrons $5 \times 10^{-14} \mathrm{~cm}^{2}$ (Ref. 15) dominates in this process. This behavior is consistent with the previously found "lifetime-killing" effect in CdTe:Ge, ${ }^{10}$ leading to a weak response of a germanium-doped CdTe crystal when used as a nuclear detector.

In conclusion, we recorded transient free carrier phase gratings using a parametric device and measured carrier transport features in light-created internal space-charge electric fields, whose strength and origin depend on deep level charge state and electron-hole competition. The experimental data at a fixed OPG wavelength confirm the applicability of the parametric device for nonresonant DFWM experiments. The given technique using a picosecond parametric device would allow the direct determination of wavelengthdependent carrier generation and recombination processes in compound crystals and, thus, optimization of their photoelectric and photorefractive properties in a required temporal and spectral range.

\section{ACKNOWLEDGMENTS}

The authors wish to thank their colleagues J. C. Launay and K. Scherbin for providing the CdTe:V and CdTe:Ge samples. K. Jarašiunas acknowledges the financial support of the Ministère de l'Enseignement Superieur et de la Recherche (France), Open Society Fund (Lithuania), and Hendrik Gerritsen for the fruitful discussions on the manuscript.

${ }^{1}$ A. Miller, D. A. B. Miller, and S. D. Smith, Adv. Phys. 30, 697 (1981).

${ }^{2}$ H. J. Eichler, P. Gunter, and D. W. Pohl, Laser-Induced Dynamic Gratings, Springer Series in Optical Science, Vol. 50 (Springer, Berlin, 1986), 211.

${ }^{3}$ J. Vaitkus, K. Jarašiūnas, E. Gaubas, L. Jonikas, and L. Subačius, IEEE J. Quantum Electron. QE-22, 1288 (1986).

${ }^{4}$ J. M. Hvam and C. Dornfeld, Growth and Optical Properties of Wide Gap II-VI Low-Dimensional Semiconductors, NATO ASI Series B Vol. 200, edited by T. C. McGill, C. M. S. Torres, and W. Gebhardt (Plenum, New York, 1989), p. 183.

${ }^{5}$ Q. Wang, R. M. Brubaker, D. D. Nolte, and M. R. Melloch, J. Opt. Soc. Am. B 9, 1626 (1992).

${ }^{6}$ D. Veleckas, K. Jarašiūnas, R. Baltramiejunas, and J. Vaitkus, Sov. Pisma J. Tekch. Fiz. 1, 708 (1975).

${ }^{7}$ Ultrashort Light Pulses, Topics in Applied Physics, Vol. 18, edited by S. L. Shapiro (Springer, Berlin, 1977), Chap. 3.

${ }^{8}$ K. Jarašiūnas, Ph. Delaye, and G. Roosen, Phys. Status Solidi B 175, 445 (1993).

${ }^{9}$ L. A. de Montmorillon et al., J. Opt. Soc. Am. B 13, 2341 (1996).

${ }^{10}$ C. Scharager, P. Siffert, P. Hoschl, P. Moravec, and M. Vanecek, Phys. Status Solidi A 66, 87 (1981).

${ }^{11}$ V. V. Matlak, E. S. Nikoniuk, A. V. Savickij, and K. D. Tovstynk, Sov. Phys. Semicond. 6, 1760 (1973).

${ }^{12}$ J. C. Launay, V. Mazoyer, M. Tapiero, J. P. Zielinger, Z. Guellil, Ph. Delaye, and G. Roosen, Appl. Phys. A: Solids Surf. 55, 33 (1992).

${ }^{13}$ G. G. Valley, J. Dubard, A. L. Smirl, and A. M. Glass, Opt. Lett. 14, 961 (1989).

${ }^{14}$ K. Jarašiūnas, Ph. Delaye, J. C. Launay, and G. Roosen, Opt. Commun. 93, 59 (1992).

${ }^{15}$ A. Savickij, Ph.D. thesis, Chernovtsy University, Ukraine, 1984. 